\title{
SYMPOSIUM ABSTRACTS
}

\section{Symposium 1: Digital Healthcare Applications \& Data Safety}

\section{Moving beyond Protection with PULSE - Using A.I. to make healthcare more affordable and accessible}

\author{
Mohd Zameen Azuan Mohd Zahari \\ Prudential Malaysia, Menara Prudential, Tun Razak Exchange, Kuala Lumpur
}

\begin{abstract}
The world has never been more connected. According to leading market intelligence firm, IDC, there will be a staggering 25 million mobile applications by 2020 with a total of 50 trillion GB of data available worldwide. Mobile applications are not only taking the world by storm, it is also redefining and transforming healthcare delivery and experience. Currently there are over 325,000 health apps available in the top app stores worldwide with more than 200 apps being added each day. Worldwide, we are seeing how digital tools are leading to better and faster healthcare and making healthcare more empowering and accessible for the people. Goldman Sachs predicts that the digital revolution could potentially save healthcare providers USD300 billion. While the adoption of digital health tools such as mobile health apps holds great promise of improving healthcare access and patient outcomes, on the flip side, the popularity of health apps is also posing enormous and unprecedented risks to consumers' privacy. PAMB is proactively transforming itself from a health payer to a holistic health manager through the implementation of PULSE, Prudential's Health Ecosystem using the Prevent-Postpone-Protect strategy. In addition, Prudential has put measures in place to safeguard consumers' privacy to ensure that data security is not compromised as we leverage on digital technology to provide consumers with instant, reliable and relevant health information at their fingertips as we empower and encourage the public to take charge of their health anytime, anywhere.
\end{abstract}

